\title{
Clear Cell Hidradenoma of the Axilla: a Case Report with Literature Review
}

Kyung Eun Cho, MD'

Eun Ju Son, MD'

Jeong-Ah Kim, MD'

Ji Hyun Youk, MD'

Eun-Kyung Kim, MD'

Jin Young Kwak, MD'

Joon Jeong, MD²
Clear cell hidradenoma is an uncommon benign skin appendageal tumor that typically involves the dermal layer of the head, face, and extremities. The breast is a rare site for this lesion, with only two documented cases, which were determined based on mammogram and sonogram findings. We present a case of clear cell hidradenoma of the axillary tail with radiological findings and a literature review.
Index terms:

Axilla mass

Hidradenoma

Ultrasonography

DOI:10.3348/kjr.2010.11.4.490

Korean J Radiol 2010; $11: 490-492$ Received December 11, 2009; accepted after revision February 4, 2010.

Departments of ${ }^{1}$ Radiology and ${ }^{2}$ Surgery, Yonsei University College of Medicine,

Seoul 135-720, Korea

Address reprint requests to: Eun Ju Son, MD, Department of Radiology, Yonsei University College of Medicine, Gangnam Severance Hospital, 712 Eonjuro, Gangnam-gu, 135-720

Seoul, Korea.

Tel. (822) 2019-3510

Fax. (822) 3462-5472

e-mail: ejsonrd@yuhs.ac
7 here are three types of sweat glands; eccrine, apocrine, and mixed (1). A clear cell hidradenoma is a rare benign sweat gland tumor which is traditionally believed to originate from the eccrine gland $(2,3)$. A clear cell hidradenoma of the breast is rare. Only two cases have been reported based on mammogram and sonogram findings $(3,4)$. Due to the rarity of this tumor, and the common removal of it without imaging studies, typical radiological findings have not yet been well established. We present a case of clear cell hidradenoma of the axillary tail with mammography and ultrasonography in a 56 year-old woman.

\section{CASE REPORT}

A 56 year-old woman presented with a solitary, palpable mass in the left axilla. This mass had been recognized for six months and displayed rapid growth. The patient had a personal history of ovarian cancer, but she had no remarkable family medical history. Upon physical examination, a 3-cm, soft mobile mass was identified in the axillary tail of the left breast. No nipple discharge or changes to the skin color were observed.

A left medio-lateral oblique (MLO) mammogram (Senograph DMR; GE Healthcare, Milwaukee, WI) showed a $2.7 \times 2.8 \mathrm{~cm}$, round, well-circumscribed, high-density mass in the left axilla (Fig. 1A). There was no combined microcalcification within or around the mass. The mass was not seen in the cranio-caudal (CC) projection. An ultrasound (iU22 unit; Philips Medical Systems, Bothell, WA) of the left breast revealed a $2.7 \times$ $2.5 \mathrm{~cm}$, oval, well-circumscribed mass with complex echogenicity in the subcutaneous layer of the left axilla (Fig. 1B). The solid portion and peripheral area of the mass showed increased vascularity following a power Doppler examination (Fig. 1C). Radiologically, this lesion was considered to be BI-RADS (Breast Imaging Reporting and Data System) category 4a. An excisional biopsy was performed at the patient's request due to the superficial location and palpability of the mass.

The biopsy specimen measured $3 \times 2.5 \times 1.5 \mathrm{~cm}$. Grossly, the mass was wellencapsulated, tan-to-pink in color, and cystic. A section of the mass showed it to have solid areas with cystic cavities containing serous fluid and white-to-tan gelatinous 


\section{Clear Cell Hidradenoma of Axilla}

material. Hematoxylin and eosin sections revealed that the tumor lobules were composed of predominantly clear cells with ductal differentiation and eosinophilic cytoplasm.

These findings were compatible with a clear cell hidradenoma (Fig. 1D).

\section{DISCUSSION}

A clear cell hidradenoma is an uncommon benign sweat gland tumor, which is traditionally known to originate

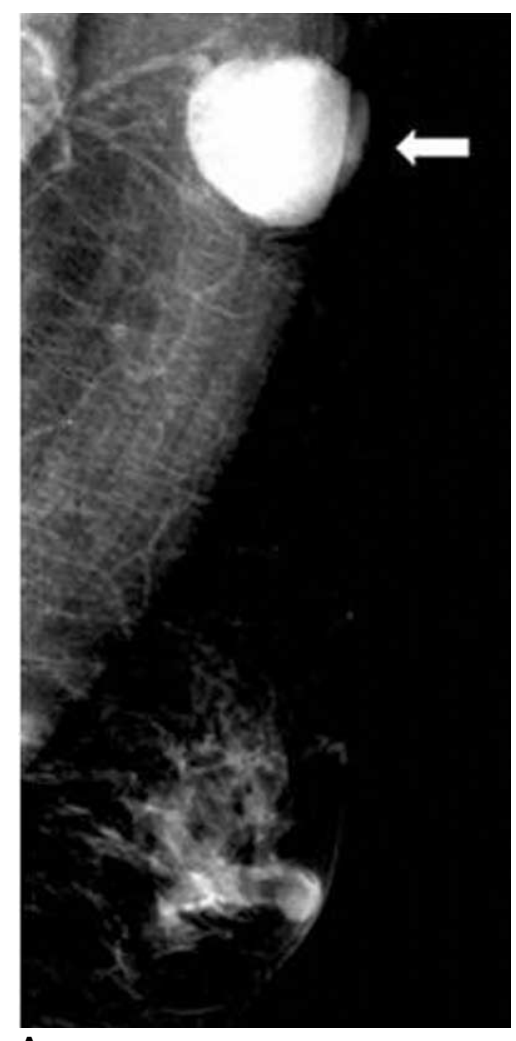

A

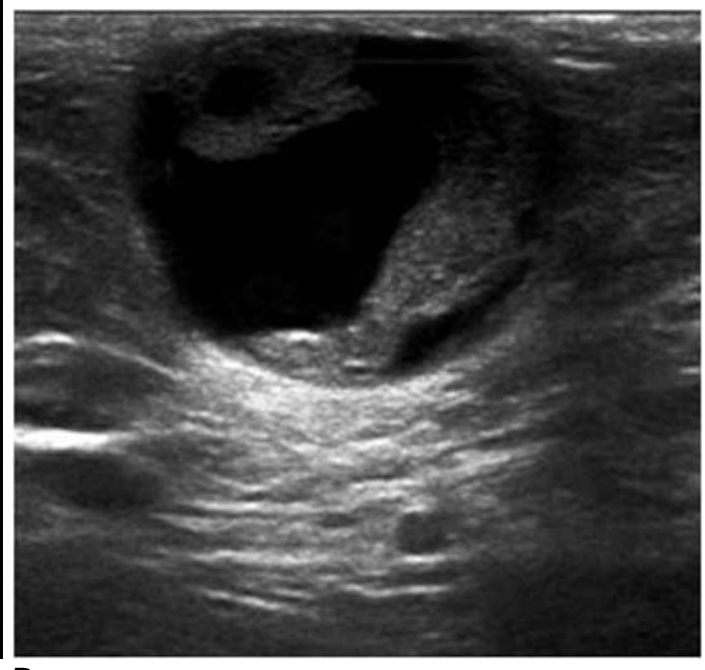

Fig. 1. 56-year-old woman with soft, mobile mass in left axillary tail for six months.

A. Medio-lateral oblique mammogram showing round, well-circumscribed, high-density mass in left axilla (white arrow).

B. Ultrasound showing oval, wellcircumscribed, complex mass with posterior acoustic enhancement in subcutaneous fat layer of left axillary tail.

C. Power Doppler study revealed increased vascularity in peripheral area and internal solid portion of mass. D. Microscopically, tumor is predominantly composed of clear cells with ductal differentiation and eosinophilic cytoplasm (Hematoxylin \& Eosin stain, $\times 400)$.

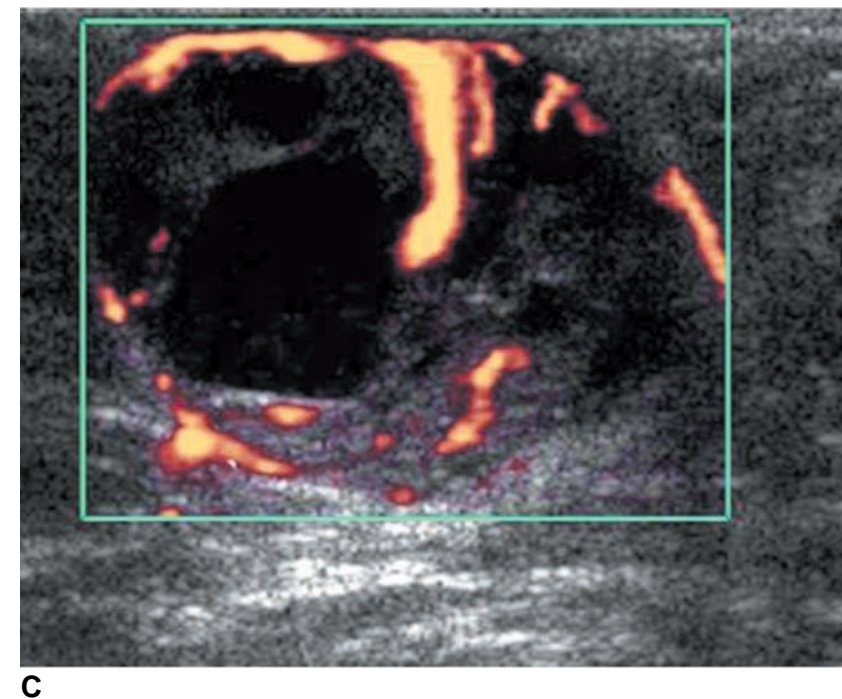

C

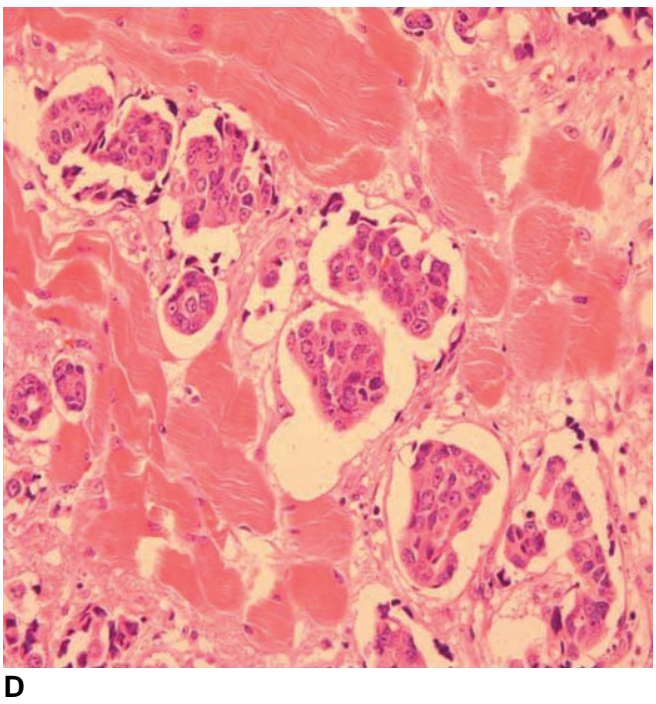




\section{Cho et al.}

in the breast parenchyma) have been diagnosed by a mammography and breast ultrasonography $(3,4)$. The case in the axillary tail presented as a well-circumscribed, highdensity mass on mammography, just as it did in our case (3). The other case, located in the breast parenchyma, presented as a focal asymmetric density (4).

An ultrasound (1-4), performed on clear cell hidradenomas present as well-defined cystic masses with mural nodules or as well-defined solid tumors with hypoechogenicity and hypervascularity. A mural nodule in a cystic lesion is also frequently highly vascular as determined by a Doppler examination. The echogenicity of the cystic portion could be complex due to a hemorrhagic component. In addition, calcifications were reported in some cases. Common features of a clear cell hidradenoma include a well-circumscribed, lobulated, subcutaneous, cystic or solid mass with low-to-intermediate signal intensity on a T1 weighted image (WI) MRI and an intermediate-to-high signal intensity upon a T2WI MRI and short tau inversion recovery image $(2,9)$. Hemorrhage and sweat gland excretion in the cystic portion may cause variable signal intensity of the fluid contents of the tumor. The mural nodule or solid portion of the tumor could show enhancement after contrast enhancement. The differential diagnosis for a clear cell hidradenoma includes primary breast cancer and papillary neoplasm of the breast $(3,4)$, hemangioma, vascular leiomyoma, lymphomas, and metastases (1). Malignant transformation, or hidradenocarcinoma, is uncommon but the differential diagnosis with a benign hidradenoma is difficult without distant metastasis or aggressive local invasion (8). Tumor remnants due to inadequate excision are a frequent cause of recurrence.
Because of this risk, an excisional biopsy with clear resection margins is necessary in order to make a final accurate diagnosis and begin the proper treatment $(2,3)$.

Although the radiological findings are non-specific, clear cell hidradenoma should be considered in the differential diagnosis when a superficially located, well-circumscribed, cystic mass with a variable solid portion and hypervascularity is encountered during a breast imaging work-up.

\section{References}

1. Jin W, Kim GY, Lew BL, Yang DM, Kim HC, Ryu JK, et al. Sonographic findings of an eccrine spiradenoma: case report and literature review. J Ultrasound Med 2008;27:813-818

2. Mullaney PJ, Becker E, Graham B, Ghazarian D, Riddell RH, Salonen DC. Benign hidradenoma: magnetic resonance and ultrasound features of two cases. Skeletal Radiol 2007;36:11851190

3. Ghai S, Bukhanov K. Eccrine acrospiroma of breast: mammographic and ultrasound findings. Clin Radiol 2004;59:1142-1144

4. Ohi Y, Umekita Y, Rai Y, Kukita T, Sagara Y, Sagara Y, et al. Clear cell hidradenoma of the breast: a case report with review of the literature. Breast Cancer 2007;14:307-311

5. Shaikh-Naidu N, Breitbart A. Eccrine spiradenoma of the upper extremity: case report and an algorithm for management. Eur J Plast Surg 2003;26:160-163

6. Revis P, Chyu J, Medenica M. Multiple eccrine spiradenoma: case report and review. J Cutan Pathol 1988;15:226-229

7. El Demellawy D, Daya D, Alowami S. Clear cell hidradenoma: an unusual vulvar tumor. Int J Gynecol Pathol 2008;27:457-460

8. Hernández-Pérez E, Cestoni-Parducci R. Nodular hidradenoma and hidradenocarcinoma. A 10-year review. J Am Acad Dermatol 1985;12:15-20

9. Han YD, Huan Y, Deng JL, Zhang YG, Zhang CH. MRI appearance of multiple eccrine spiradenoma. Br J Radiol 2007;80:E27E29 